\title{
Genomic stability of genotyping markers in Chlamydia trachomatis
}

\begin{abstract}
João Paulo Gomes*
Department of Infectious Diseases, National Institute of Health, Lisbon, Portugal

*Correspondence: j.paulo.gomes@insa.min-saude.pt
\end{abstract}

\section{A commentary on}

Genotyping markers used for multi locus VNTR analysis with ompA (MLVA-ompA) and multi sequence typing retain stability in Chlamydia trachomatis

by Labiran, C., Clarke, I. N., Cutcliffe, L. T., Wang, Y., Skilton, R. J., Persson, K., Bjartling, C., Herrmann, B., Christerson, L., and Marsh, P. (2012). Front. Cell. Infect. Microbiol. 2:68. doi: 10.3389/fcimb.2012.00068

Chlamydia trachomatis is an obligate intracellular human pathogen that infects columnar epithelial cells of ocular or genital mucosae, and mononuclear phagocytes. More than 50 fully sequenced genomes are publically available (Harris et al., 2012), and despite low polymorphic character when compared with most bacteria, these data allow a better understanding of C. trachomatis chromosomal dynamics. Indeed, it revealed not only the chromosome regions more prone to genetic mutations, but also that recombination is highly chromosome dispersed, although the frequency and relative weight of recombination and mutation events undoubtedly posits $C$. trachomatis as a low recombining bacterium (Joseph et al., 2011; Ferreira et al., 2012).

In order to understand the epidemiology and pathogenesis of the highly prevalent chlamydial diseases, the classification of clinical isolates has been a priority of researchers for decades. However, the traditional typing system, which is based on the polymorphism of the gene (ompA) encoding the major outer membrane protein (Yuan et al., 1989) has been jeopardized in the last years as it does not group the isolated strains according to their cellappetence or virulence. Thus, with the progressive release of sequenced genomes, several typing systems have been developed (Klint et al., 2007; Pannekoek et al., 2008; Pedersen et al., 2008; Dean et al., 2009; Bom et al., 2011), although none of them is consensual due to the dissimilar genetic characteristics or discriminatory power of the loci enrolled in each of those.

Although it is assumed that the typing schemes should ideally ensure that the chosen loci are stable over the course of evolution, this issue is of difficult assessment. Labiran et al. (2012) have now presented a study where they have assessed the stability of some regions of the $C$. trachomatis genome by using innovate approaches. They have used the genetic markers from the described typing schemes that provide the highest Simpson index of diversity: the multisequence typing (MST) system described by Klint et al. (2007) and the multi locus variable number tandem repeat (VNTR) analysis (MLVA-ompA) described by Pedersen et al. (2008). Labiran et al. (2012) have initially evaluated the stability of those genetic markers through the first stages of adaptation of C. trachomatis to cell culture. This is interesting as the first attempt to study mutational patterns over adaptation to cell culture had been performed back in 1998 (Stothard et al., 1998), by studying solely the stability of ompA over 20 in vitro passages. Now, by analyzing three VNTR besides ompA, the authors undoubtedly increase the chances of observing genetic alterations, as these loci possess a high discriminatory power. Furthermore, as the repeated sequences of VNTR loci are generated as a consequence of an increased error rate during DNA replication at these regions, they are hypothetically more prone to additional variation. In this first approach, the authors found no alterations in those genomic regions by studying seven clinical isolates over eight passages. The initial inoculations were properly done from primary swabs in order to avoid previous contact of the strains with the in vitro system. Interestingly, the eight passages represent about 16 days in culture aiming to reflect the in vivo estimated period for appearance of symptoms for C. trachomatis infection of both genitalia and conjunctivae (Black, 1997). There is no doubt that there is a radical change of environmental conditions when transiting from the in vivo to the in vitro system, mostly because the latter lacks immune system, competing flora, $\mathrm{pH}$ alterations, hormonal fluctuations, etc. Nevertheless, although the parallelism between these two scenarios remains speculative for these reasons, the impossibility to perform this evaluation in vivo and under controlled conditions makes this an interesting approach.

In a second stage, the authors went even further by studying the "long-term" genome stability under influence of in vitro environmental conditions. To achieve this, they used the MLVA-ompA and also the fivelocus MST system to monitor the stability of the enrolled loci throughout 72 passages in tissue culture. The coverage and the runtime of this approach make it an unprecedented evaluation of genome stability. In this study Labiran et al. (2012) used the so called "Swedish new variant," a C. trachomatis isolate that, besides the higher number of pseudogenes than most sequenced genital isolates (Jeffrey et al., 2010), shows both a deletion and duplication within the plasmid, which are genomic changes that had never been described before (Unemo et al., 2010). These kind of genomic alterations are infrequent as C. trachomatis presents a nearly identical core and pan genome and a DNA sequence similarity of $>98 \%$ (Harris et al., 2012). So, this isolate could hypothetically be considered as genomically unstable, making this a valuable strain to test stability over a long time in vitro passage. Similarly to the previous approach, no genetic changes were observed throughout the long-term in vitro maintenance, suggesting that the MLVA-ompA and MST systems are, on a genetic stability basis, highly accurate for both short-term (such as partner tracing) and long-term molecular epidemiology.

Moreover, the results from the Labiran et al. (2012) study indirectly bring to light a major controversy in the field, which is the uncertainty of whether the prototype 
strains that have been used worldwide leading to thousands of papers, accurately represent current circulating clinical strains. In fact, the former are laboratory adapted for decades but at this stage one cannot know the extent of genomic alterations that may occur due to laboratory adaptation. On one hand, the results from Labiran et al. (2012) point to specific genomic stability, which, together with the $C$. trachomatis transmission bottlenecks (Andersson and Hughes, 1996) and the high doubling time of 2.5-4 h (Borges et al., 2010), disfavors genetic variability. On the other hand, it is known that some loci are prone to generate multi-alleles, such as the putative virulence factor CT135 (Sturdevant et al., 2010), and that Chlamydia undergoes adaptive changes when subjected to specific environmental alterations (Kutlin et al., 2005; Suchland et al., 2005). Ultimately, unless the entire genome is analyzed (at the stage of the strain isolation and after long-term in vitro passage), the controversy will certainly remain.

\section{REFERENCES}

Andersson, D. I., and Hughes, D. (1996). Muller's ratchet decreases fitness of a DNA-based microbe. Proc. Natl. Acad. Sci. U.S.A. 93, 906-907.

Black, C. M. (1997). Current methods of laboratory diagnosis of Chlamydia trachomatis infections. Clin. Microbiol. Rev. 10, 160-184.

Bom, R. J., Christerson, L., Schim Van Der Loeff, M. F., Coutinho, R. A., Herrmann, B., and Bruisten, S. M. (2011). Evaluation of high-resolution typing methods for Chlamydia trachomatis in samples from heterosexual couples. J. Clin. Microbiol. 49, 2844-2853.

Borges, V., Ferreira, R., Nunes, A., Nogueira, P., Borrego, M. J., and Gomes, J.P. (2010). Normalization strategies for real-time expression data in Chlamydia trachomatis. J. Microbiol. Methods 82, 256-264.

Dean, D., Bruno, W. J., Wan, R., Gomes, J. P., Devignot, S., Mehari, T., De Vries, H. J. C., Morre, S. A., Myers,
G., Read, T. D., and Spratt, B. G. (2009). Predicting phenotype and emerging strains among Chlamydia trachomatis infections. Emerging Infect. Dis. 15, 1385-1394.

Ferreira, R., Borges, V., Nunes, A., Nogueira, P. J., Borrego, M. J., and Gomes, J. P. (2012). Impact of loci nature on estimating recombination and mutation rates in Chlamydia trachomatis. G3 (Bethesda). 2:761-768. doi: $10.1534 / \mathrm{g} 3.112 .002923$

Harris, S. R., Clarke, I. N., Seth-Smith, H. M., Solomon, A. W., Cutcliffe, L. T., Marsh, P., Skilton, R. J., Holland, M. J., Mabey, D., Peeling, R. W., Lewis, D. A., Spratt, B. G., Unemo, M., Persson, K., Bjartling, C., Brunham, R., de Vries, H. J., Morré, S. A., Speksnijder, A., Bébéar, C. M., Clerc, M., de Barbeyrac, B., Parkhill, J., and Thomson, N. R. (2012). Whole-genome analysis of diverse Chlamydia trachomatis strains identifies phylogenetic relationships masked by current clinical typing. Nat. Genet. 44, 413-419.

Jeffrey, B. M., Suchland, R. J., Quinn, K. L., Davidson, J. R., Stamm, W. E., and Rockey, D. D. (2010). Genome sequencing of recent clinical Chlamydia trachomatis strains identifies loci associated with tissue tropism and regions of apparent recombination. Infect. Immun. 78, 2544-2553.

Joseph, S. J., Didelot, X., Gandhi, K., Dean, D., and Read, T. D. (2011). Interplay of recombination and selection in the genomes of Chlamydia trachomatis. Biol. Direct 6, 28.

Klint, M., Fuxelius, H. H., Goldkuhl, R. R., Skarin, H., Rutemark, C., Andersson, S. G. E., Persson, K., and Herrmann, B. (2007). High-resolution genotyping of Chlamydia trachomatisstrains by multilocus sequence analysis. J. Clin. Microbiol. 45, 1410-1414.

Kutlin, A., Kohlhoff, S., Roblin, P., Hammerschlag, M. R., and Riska, P. (2005). Emergence of resistance to rifampin and rifalazil in Chlamydophila pneumoniae and Chlamydia trachomatis. Antimicrob. Agents Chemother. 49, 903-907.

Labiran, C., Clarke, I. N., Cutcliffe, L. T., Wang, Y., Skilton, R. J., Persson, K., Bjartling, C., Herrmann, B., Christerson, L., and Marsh, P. (2012). Genotyping markers used for multi locus VNTR analysis with ompA (MLVA-ompA) and multi sequence typing retain stability in Chlamydia trachomatis. Front. Cell. Infect. Microbiol. 2:68. doi: 10.3389/fcimb.2012.00068

Pannekoek, Y., Morelli, G., Kusecek, B., Morre, S. A., Ossewaarde, J. M., Langerak, A. A., and Van Der Ende,
A. (2008). Multi locus sequence of Chlamydiales: clonal groupings within the obligate intracellular bacteria Chlamydia trachomatis. BMC Microbiol. 8, 42. doi: 10.1186/1471-2180-8-42

Pedersen, L. N., Podenphant, L., and Moller, J. K. (2008). Highly discriminative genotyping of Chlamydia trachomatis using omp1 and a set of variable number tandem repeats. Clin. Microbiol. Infect. 14, 644-652.

Stothard, D. R., Van Der Pol, B., Smith, N. J., and Jones, R. B. (1998). Effect of serial passage in tissue culture on sequence of omp1 from Chlamydia trachomatis clinical isolates. J. Clin. Microbiol. 36, 3686-3688.

Sturdevant, G. L., Kari, L., Gardner, D. J., OlivaresZavaleta, N., Randall, L. B., Whitmire, W.M., Carlson, J. H., Goheen, M. M., Selleck, E. M., Martens, C., and Caldwell, H. D. (2010). Frameshift mutations in a single novel virulence factor alter the in vivo pathogenicity of Chlamydia trachomatis for the female murine genital tract. Infect. Immun. 78, 3660-3668.

Suchland, R. J., Bourillon, A., Denamur, E., Stamm, W. E., and Rothstein, D. M. (2005). Rifampin-resistant RNA polymerase mutants of Chlamydia trachomatis remain susceptible to the ansamycin rifalazil. Antimicrob. Agents Chemother. 49, 1120-1126.

Unemo, M., Seth-Smith, H. M., Cutcliffe, L. T., Skilton, R. J., Barlow, D., Goulding, D., Persson, K., Harris, S. R., Kelly, A., Bjartling, C., Fredlund, H., Olcén, P., Thomson, N. R., and Clarke, I. N. (2010). The Swedish new variant of Chlamydia trachomatis: genome sequence, morphology, cell tropism and phenotypic characterization. Microbiology 156, 1394-1404.

Yuan, Y., Zhang, Y. X., Watkins, N. G., and Caldwell, H. D. (1989). Nucleotide and deduced amino acid sequences for the four variable domains of the major outer membrane proteins of the 15 Chlamydia trachomatis serovars. Infect. Immun. 57, 1040-1049.

Received: 14 May 2012; accepted: 18 May 2012; published online: 06 June 2012.

Citation: Gomes JP (2012) Genomic stability of genotyping markers in Chlamydia trachomatis. Front. Cell. Inf. Microbio. 2:77. doi: 10.3389/fcimb.2012.00077

Copyright $(\odot 2012$ Gomes. This is an open-access article distributed under the terms of the Creative Commons Attribution Non Commercial License, which permits non-commercial use, distribution, and reproduction in other forums, provided the original authors and source are credited. 